\title{
A Growth-Inhibitory Effect on Shigella dysenteriae which Occurs with some Batches of Nutrient Agar and is Associated with the Production of Peroxide
}

\author{
By H. PROOM, A. J. WOIWOD, JOAN M. BARNES \\ AND W. G. ORBELL \\ The Wellcome Research Laboratories, Beckenham, Kent
}

\begin{abstract}
SUMMARY: Certain batches of nutrient agar were inhibitory to small inocula of Shigella dysenteriae. The inhibitory effect was abolished by catalase and by manganese dioxide. It occurred only with surface cultures incubated aerobically; poured plates or plates incubated anaerobically showed no inhibition. The inhibitor was a peroxide formed during the heating of nutrient broth and agar; it was present in minimal inhibitory concentrations since dilution with one to four volumes of saline agar removed the inhibition. It is suggested that some nutrient agars contain material which, on exposure to air, forms inhibitory concentrations of peroxide in the surface layer.
\end{abstract}

During the examination of papain digest broths for their growth-promoting properties one of us (H. P.) made the following observation. Shigella dysenteriae, strain CN191 from the Wellcome Bacterial Collection, was used to test two types of broth-broth (a) an undiluted papain digest of horse muscle containing about $7 \cdot 5 \mathrm{~g}$. total N/1., and broth $(b)$ which consisted of broth (a) diluted with an equal volume of $0.5 \%$ saline. The smallest inoculum giving growth was the same with both broths. The broths were then converted into nutrient agars by the addition of $2.0 \%$ Japanese agar. The growth from a heavy surface inoculum was about the same with both nutrient agars, but with dilute inocula more colonies grew from the agar made with the diluted broth than on the agar from the undiluted broth. Moreover, progressive dilution of the nutrient agar made from broth $(a)$ with saline agar increased the number of colonies growing from dilute inocula. No such difference was observed when silica gel was substituted for agar as the solidifying agent.

The failure of the test organism to grow thus appeared to be due to some inhibitor. The inhibitor was not present in the broth, since the smallest inoculum which grew was the same with both broths. Similarly, the inhibitor was not present in the agar itself, since dilution of a 'bad' nutrient agar with saline agar removed the inhibition. The effect was also observed during the development of tests for standardizing bacteriological media. It was subjected to systematic study and the results are reported below.

\section{EXPERIMENTAL}

Preparation of the nutrient agars

Nutrient broth was prepared by extracting fresh minced horse muscle with hot water in the proportion of $c .700 \mathrm{~g}$. muscle to 11 . water. The meat particles were removed by filtration and to the extract was added a concentrated 
peptone solution equivalent to an additional total nitrogen content of $1.5 \mathrm{~g} . / 1$. Then $0 \cdot 125 \% \mathrm{CaCl}_{2}$ was added and the $\mathrm{pH}$ adjusted to $7 \cdot 8$. $\mathrm{NaCl}$ was added to a final concentration of $0.5 \%$ and the mixture boiled, filtered and sterilized by Seitz filtration. The total nitrogen content of this broth was about 3.0 g./1. The peptone used was prepared by digesting horse muscle at pH 5.8 with papain for $2 \mathrm{hr}$. at $60^{\circ}$. The mixture was then boiled and sterilized by filtration. The total nitrogen content of this concentrated peptone was about 14 g./1. Nutrient agar was prepared by adding $1.25 \%$ New Zealand agar (Davis Gelatin (N.Z.) Ltd., Christchurch, New Zealand) to nutrient broth. The nutrient agar was filtered hot and sterilized by autoclaving.

\section{Comparison of growth on different batches of nutrient agar}

A convenient test for comparing the growth on different batches of solid medium was the determination of the viable count of a bacterial suspension by the Miles \& Misra (1938) technique, using an arbitrarily selected batch of medium as a standard for comparison. An overnight culture on nutrient agar of strain CN 191 was emulsified in Ringer's solution and diluted to an opacity corresponding to Brown's opacity tube No. 3 (Burroughs, Wellcome Ltd.), i.e. approximately $2000 \times 10^{6}$ organisms $/ \mathrm{ml}$. Single drops, from a $0.02 \mathrm{ml}$. dropping pipette, of ten-fold serial dilutions of this suspension were dropped on agar plates and incubated in the usual manner. Four dilutions were used for each plate.

\section{The difference between good and bad nutrient agars}

Although all batches of nutrient agar used were prepared by the same standard method, two types were encountered. A 'good' nutrient agar gave confluent growth with an inoculum of a dilution of $10^{-5}$ of the test suspension, about 50 colonies at a dilution of $10^{-6}$, and an occasional colony at $10^{-7}$. On the other hand, a 'bad' nutrient agar gave confluent growth with a dilution of $10^{-1}$ and no growth with the subsequent dilutions. The two types of nutrient agar were indistinguishable when tested against Escherichia coli or Staphylococcus aureus, the bad nutrient agar having no inhibitory action against these organisms. This effect was shown to be a species effect and was not confined to the particular strain of $S h$. dysenteriae used. Six other strains of $S h$. dysenteriae were inhibited by the bad nutrient agar but no strains of the species Sh. paradysenteriae, Esch. coli, or Staph. aureus were affected.

Dilution of broth with saline before the preparation of nutrient agar or the dilution of a bad nutrient agar with saline agar eliminated the inhibitory effect. A typical protocol is given in Table 1, showing that relatively slight (1/3) dilution with saline agar was sufficient to remove the inhibition. Dilution with one or two volumes of saline agar was always sufficient to overcome any inhibitory effect.

\section{Effect of various substances on the inhibition}

A systematic search was made for substances which might neutralize the inhibitory effect of bad nutrient agars. A large number of growth factors, separately or together, casein acid-hydrolysate, starch, or thiolacetic acid 
Table 1. The effect of dilution of good and bad nutrient agars with saline agar on the growth of Shigella dysenteriae from small inocula

Growth from 0.02 ml. inoculum of dilution

\begin{tabular}{lcccccccc}
\multicolumn{1}{c}{ Medium } & $10^{-1}$ & $10^{-2}$ & $10^{-8}$ & $10^{-4}$ & $10^{-5}$ & $10^{-6}$ & $10^{-7}$ \\
Good agar (batch M 259/48) & + & + & + & + & + & 25 & 3 \\
Good agar (3 vol.) + saline agar (1 vol.) & + & + & + & + & + & 22 & 2 \\
Good agar (1 vol.) + saline agar (1 vol.) & + & + & + & + & + & 28 & 1 \\
Good agar (1 vol.) + saline agar (3 vol.) & + & + & + & + & + & 46 & 6 \\
Bad agar (batch M319/48) & + & - & - & - & - & - & - \\
Bad agar (3 vol.) + saline agar (1 vol.) & + & - & - & - & - & - & - \\
Bad agar (1 vol.) + saline agar (1 vol.) & + & + & + & 96 & 8 & - & - \\
Bad agar (1 vol.) + saline agar (3 vol.) & + & + & + & + & + & 26 & 3
\end{tabular}

Undiluted inoculum $=c$. $2 \times 10^{\circ}$ organisms $/ \mathrm{ml}$. $+=$ confluent growth or numerous colonies. Numerals $=$ no. of colonies.

Table 2. Neutralization of the inhibitory effect of a bad nutrient agar on Shigella dysenteriae by dilutions of purified catalase

Growth from $0.02 \mathrm{ml}$. inoculum of dilution

Medium
Good agar (batch M 259/48)
Bad agar (batch $\mathbf{M 4 0 / 4 8 )}$
$15 \mathrm{ml}$. bad agar $+1 \mathrm{ml}$. $0 \cdot 85 \%$ saline
$15 \mathrm{ml}$. bad agar $+1 \mathrm{ml}$. catalase solution diluted $10^{-2}$
$15 \mathrm{ml}$ bad agar $+1 \mathrm{ml}$. catalase solution diluted $10^{-4}$
$15 \mathrm{ml}$. bad agar $+1 \mathrm{ml}$. catalase solution diluted $10^{-6}$
$15 \mathrm{ml}$. bad agar $+1 \mathrm{ml}$. catalase solution diluted $10^{-8}$
$15 \mathrm{ml}$ bad agar $+1 \mathrm{ml}$. catalase solution diluted $10^{-10}$

$\begin{array}{cccc}10^{-1} & 10^{-5} & 10^{-6} & 10^{-7} \\ + & + & 37 & 3 \\ - & - & - & - \\ - & - & - & - \\ + & + & 48 & 6 \\ + & + & 51 & 9 \\ + & + & 18 & - \\ + & 88 & 8 & - \\ + & 17 & 3 & -\end{array}$

Undiluted inoculum $=c .2 \times 10^{\circ}$ organisms $/ \mathrm{ml}$. $+=$ confluent growth or numerous colonies. Numerals $=$ no. of colonies.

$(0.01 \%$ incorporated in the medium) did not neutralize the inhibitor. The inhibitory effect was, however, abolished by fresh blood, some vegetable extracts such as extract of horse radish, a purified catalase preparation and manganese dioxide, but not by salts of manganese. With the exception of manganese dioxide, the ability of these substances to neutralize the inhibitor was destroyed by heat. Table 2 shows the neutralization of the inhibitory effect by purified catalase (kindly supplied by Dr D. Herbert).

The abolition of the inhibition by high dilutions of catalase, but not by heated catalase, and also by manganese dioxide, but not by salts of manganese, can be regarded as reasonable proof that the inhibition was due to a peroxide. This agrees with the observation that catalase-producing organisms such as Sh. paradysenteriae, Esch. coli, or Staph. aureus were not inhibited by bad nutrient agars; Sh. dysenteriae does not produce catalase.

Manganese dioxide destroyed the inhibitory effect of a bad nutrient agar. This was shown either by incorporating a little solid $\mathrm{MnO}_{2}$ in the medium (Table 3) or by placing a little $\mathrm{MnO}_{2}$ on the surface of an agar plate. In the latter case, when the surface of a plate was inoculated with a dilute inoculum, 
colonies of Sh. dysenteriae grew in the immediate vicinity of the $\mathrm{MnO}_{2}$ particles but not elsewhere. Table 3 also shows that when a bad nutrient agar containing $\mathrm{MnO}_{2}$ was filtered the filtrate was no longer inhibitory. However, when this filtered agar was autoclaved it again became inhibitory. This indicated that the inhibitor or its precursor was formed, or its formation was rapidly accelerated, during the heating of this nutrient agar.

Table 3. The effect of $\mathrm{MnO}_{2}$ on the inhibitory action of a bad nutrient agar on Shigella dysenteriae

\begin{tabular}{|c|c|c|c|c|}
\hline \multirow[b]{2}{*}{ Medium } & \multicolumn{4}{|c|}{$\begin{array}{c}\text { Growth of } S h . \text { dysenteriae } \\
\text { with } 0.02 \mathrm{ml} \text {. inoculum } \\
\text { of dilution }\end{array}$} \\
\hline & $10^{-4}$ & $10^{-5}$ & $10^{-6}$ & $10^{-7}$ \\
\hline Good agar (batch 259/48) & + & 130 & 13 & 1 \\
\hline Good agar $+\mathrm{MnO}_{2}(1 \mathrm{~g} . / 1)$. & + & 210 & 14 & 4 \\
\hline Bad agar (batch 840/48) & 一 & - & - & - \\
\hline Bad agar + 1 g./1. $\mathrm{MnO}_{2}$ & + & 200 & 16 & $\mathbf{3}$ \\
\hline Bad agar +1 g./l. $\mathrm{MnO}_{2}$ and filtered* & + & 79 & 12 & $\mathbf{1}$ \\
\hline Bad agar +1 g./l. $\mathrm{MnO}_{2}$ filtered and autoclaved $\dagger$ & 6 & - & - & - \\
\hline Bad agar + 0.001 \% $\mathrm{MnSO}_{4}$ & - & - & - & - \\
\hline
\end{tabular}

* Fịltered through Seitz pads.

$\uparrow$ Autoclaved $15 \mathrm{~min}$. at $15 \mathrm{lb}$.

Undiluted inoculum $=c .2 \times 10^{\circ} \mathrm{organisms} / \mathrm{ml}$. $+=$ confluent growth or numerous colonies. Numerals $=$ no. of colonies.

It was possible to convert a good nutrient agar into a bad nutrient agar by continuous autoclaving. The degree of autoclaving necessary to do this varied from batch to batch. But here it was necessary to distinguish between a bad nutrient agar of the type under investigation and nutrient agars which by continued heating had lost growth-promoting properties for other species. This was done by noting whether the inhibition was removed by catalase and whether the nutrient agar was still capable of growing Staph. aureus.

Attempts made to extract the inhibitor were uniformly unsuccessful. Saline extracts of bad agars were made and tested in parallel with nutrient broth. The smallest inoculum to grow was the same in both cases. When these extracts were reconverted into nutrient agar by steaming with $1.25 \%$ agar they were non-inhibitory. When the dilutions of test inoculum were made in extracts from bad nutrient agars and tested on a good nutrient agar no inhibition was observed. These results would confirm that the peroxide was present only in minimal inhibitory amounts. Chemical tests for peroxide in bad nutrient agars were negative.

The smallest inocula to grow on good and bad nutrient agars were compared by the poured plate method as well as by the surface method of Miles \& Misra. No inhibition was shown with a bad nutrient agar as a poured plate. Good and bad nutrient agars were then compared when surface-inoculated plates were incubated anaerobically; under anaerobic conditions a bad nutrient agar was no longer inhibitory. Sandwich plates were made with various combinations of good and bad nutrient agars. Table 4 illustrates the results obtained. The 
first layer was poured, dried and inoculated, and after the inoculum had dried the second layer of agar was then poured on top. When the two layers were both of bad nutrient agar some inhibition was obtained, but with all other combinations little inhibition was observed.

Table 4. The effect of sandwich plates and anaerobiosis on the growth of Shigella dysenteriae on good and bad nutrient agars

\begin{tabular}{|c|c|c|c|c|}
\hline \multirow[b]{2}{*}{ Medium } & \multicolumn{4}{|c|}{$\begin{array}{c}\text { Growth of Sh. dysenteriae } \\
\text { with inoculum of } \\
\text { dilution }\end{array}$} \\
\hline & $10^{-4}$ & $10^{-5}$ & $10^{-6}$ & $10^{-7}$ \\
\hline Good agar (batch M 402/48) incubated aerobically & + & + & 19 & 6 \\
\hline Bad agar (batch $M 605 / 58$ ) incubated aerobically & $\longrightarrow$ & 一 & - & - \\
\hline Good agar (M402/48) incubated anaerobically & + & + & 25 & $\mathbf{1}$ \\
\hline Bad agar (M 605/48) incubated anaerobically & + & + & 19 & 3 \\
\hline Sandwich plates: good agar upon bad agar & + & + & 18 & 2 \\
\hline Sandwich plates: bad agar upon good agar & + & 31 & 9 & 1 \\
\hline Sandwich plates : bad agar upon bad agar & 19 & 20 & 4 & $-\infty$ \\
\hline Sandwich plates: good agar upon good agar & + & + & 21 & 2 \\
\hline
\end{tabular}

Undiluted inoculum $=c .2 \times 10^{9}$ organisms $/ \mathrm{ml}$. $+=$ confluent growth or numerous colonies. Numerals $=$ no. of colonies.

The surface of some bad agar plates was removed by careful scraping or by slicing off the top layer, and then inoculated on the new surface by the usual technique. Inhibition was again observed, thus showing either that the inhibition was not confined to the surface layer or that a new inhibitor was formed on exposure of the fresh surface to air.

Attempts were made to simulate bad nutrient agar by the addition of hydrogen peroxide to good nutrient agar. In a number of experiments whenever an inhibitory concentration of peroxide was present in the medium, growth on both surface-inoculated and poured plates was inhibited. It was not found possible to produce only surface inhibition by addition of hydrogen peroxide.

\section{DISCUSSION}

The complex nature of nutrient agar and the small amount of peroxide that must be present makes the identification of the inhibitor, or of the mechanism leading to its formation, extremely difficult. The removal of the inhibition by catalase or manganese dioxide may be accepted as reasonable proof that the inhibitor is indeed a peroxide. This peroxide is present in amounts which cannot be detected by the usual chemical tests. Since dilution with one to four volumes of saline agar usually abolishes the inhibition, the peroxide present must be near the limit of its minimal bacteriostatic concentration. Various authors have shown that exposure of nutrient media to sunlight, ultraviolet radiation or X-rays may produce an inhibitory medium. Burnet (1925) demonstrated that peroxide was formed in nutrient agar exposed to sunlight. Wyss, Clark, Haas \& Stone (1948) showed that organic peroxides 
were formed in broth irradiated with ultraviolet light. Blank \& Amold (1935) found that irradiated carbohydrates or agar were inhibitory to Bacillus subtilis when incorporated into nutrient agar. These results differ in several respects from those described here. In the experiments of Burnet (1925) and Wyss et al. (1948) the concentration of peroxide formed was sufficient to inhibit a catalaseproducing organism such as Staph. aureus, whereas in our experiments the concentration of peroxide formed was only sufficient to inhibit a non-catalaseproducing organism. However, on one occasion, prolonged heating of a batch of bad nutrient agar produced a very dark medium which inhibited Staph. aureus and Sh. dysenteriae with both surface and poured plate cultures. This inhibition was overcome by catalase. In this respect our results may be regarded as quantitatively different from those quoted. Another difference is the mechanism by which peroxide is formed. In our experiments irradiation was not a significant factor. The peroxide was formed by reaction between material present in some broths and material normally present in agar. The production of peroxide was associated with heat and the presence of atmospheric oxygen. In fact, it was found possible to produce a bad agar by bubbling oxygen through melted good nutrient agar maintained at $47^{\circ}$ in a water bath. In this case the agar became lighter in colour, demonstrating that the production of peroxide is not necessarily associated with the darkening of nutrient agar which occurs on prolonged heating. On the available evidence it seems that when some nutrient broths are heated with agar-agar some material is formed which on exposure to atmospheric oxygen readily produces an inhibitory concentration of peroxide. This material is distributed throughout the medium, but the peroxide is apparently confined to the surface layer. It is unlikely that peroxide is present throughout the medium since, when inhibitory concentrations of peroxide are added to good nutrient agar, it is inhibitory when inoculated by either the pour-plate or surface technique. Moreover, it may seem unlikely that peroxide would not be inhibitory to $S h$. dysenteriae when growing under microaerophilic (or anaerobic) conditions. However, it is not possible to dismiss entirely the possibility that these low concentrations of peroxide may be distributed uniformly throughout the medium and that only the strictly aerobic metabolism of $S h$. dysenteriae is affected.

These results are of some practical interest in connexion with the preparation of nutrient agars, particularly for surface growth from small inocula. They also have a more general appeal because of the interest which is being shown in the study of growth inhibitors and the conditions under which they arise. Peroxide is another example of such an inhibitor and the destruction of peroxide may be another function of the blood used in some bacteriological media. Since peroxide formation is always a possibility when the surface of organic material is exposed to the atmosphere the production of catalase will be an advantage to aerobic bacteria. 


\section{REFERENCES}

Brank, H. I. \& Annold, W. (1935). The inhibition of growth of Bacillus subtilis by ultraviolet irradiated carbohydrates. J. Bact. 33, 507.

Burnet, F. M. (1925). Hydrogen peroxides and bacterial growth. Aust. J. exp. Biol. med. Sci. $2,77$.

Mrues, A. A. \& Misra, S. S. (1938). Estimation of bactericidal power of blood. J. Hyg., Camb., 38, 732.

Wyss, O., Ceatix, J. B., HAAs, F. \& STONE, W. S. (1948). The role of peroxide in the biologicinl effects of irradiated broth. J. Bact. 56, 51 .

(Received 2 November 1949) 